\title{
Can we make quantitative predictions for relative yield with incomplete knowledge of model parameters?
}

\author{
H. Fort \\ Institute of Physics, Faculty of Science, Universidad de la República, Iguá 4225, Montevideo 11400 Uruguay. \\ Phone: +598-25258624. E-mail: hugo@fisica.edu.uy
}

Keywords: Biodiversity-ecosystem functioning experiments, Quantitative Lotka-Volterra competition theory.

\begin{abstract}
A main limitation in community ecology for making quantitative predictions on species abundances is often an incomplete knowledge of the parameters of the population dynamics models. The simplest linear Lotka-Volterra competition equations (LLVCE) for $S$ species require $S^{2}$ parameters to solve for equilibrium abundances. The same order of experiments are required to estimate these parameters, namely the carrying capacities (from monoculture experiments) and the competition coefficients (from biculture or pairwise experiments in addition to monoculture ones). For communities with large species richness $S$ it is practically impossible to perform all these experiments. Therefore, with an incomplete knowledge of model parameters it seems more reasonable to attempt to predict aggregated or mean quantities, defined for the whole community of competing species, rather than making more detailed predictions, like the abundance of each species. Here we test a recently derived analytical approximation for predicting the Relative Yield Total (RYT) and the Mean Relative Yield (MRY) as functions of the mean value of the interspecific competition matrix $a$ and the species richness $S$. These formulae with only a fraction of the model parameters, are able to predict accurately empirical measurements covering a wide variety of taxa such as algae, vascular plants, protozoa. We discuss the dependence of these global community quantities on the species richness and the intensity of competition and possible applications are pointed out.
\end{abstract}

\section{Introduction}

Two main properties of an ecological community are its productivity (e.g., its total biomass or yield) and its diversity, understanding the relationship between them is crucial both in agriculture science, for productivity optimization (Fort et al. 2017, Halty et al. 2017), and in conservation ecology, for protecting endangering species and ecological communities. Competition plays a key role in determining the how the total biomass of a community is partitioned among species. The classical theoretical framework for describing the competition among $S$ species in its simplest form is provided by the linear Lotka-Volterra competition equations (LLVCE) (Lotka 1925, Volterra 1926). A convenient way of writing the equations for the species yields at equilibrium $Y_{i}$-independent of how the yield is measured in different communities (population density, biomass density or biovol) - is in terms of the relative yields $y_{i}=Y_{i} / K_{i}$, i.e., the species yield in mixture normalized by its yield in monoculture or carrying capacity $K_{i}$, (Fort 2018):

$\sum_{j=1}^{S} a_{i j} y_{j}=1, \quad i=1, \ldots, S$,

where $a_{i j}$ is the coefficient of competition of species $j$ over species $i\left(a_{i i}=1\right)$.

A main difficulty for obtaining the $y_{i}$ from (1) is that it involves the $S^{2}$ parameters $\left\{a_{i j}\right\}$ and thus at least the same number of experimental treatments is required to estimate them. Hence, for large values of $S$, only a fraction of these experiments is commonly carried out and consequently we have to deal with an incomplete knowledge of the parameters required to solve (1). This could explain why the focus of Lotka-Volterra competition theory has been almost exclusively on qualitative issues, like community stability and species coexistence, rather than on making quantitative predictions to be tested against experimental data, like relative species abundance in equilibrium (Hubbel 2002).

Here we show that, even with an incomplete knowledge of the LLVCE parameters, quantitative predictions are still possible if rather than attempting to predict the individual species yields, we aim at predicting aggregate or mean community quantities. A first example of aggregate quantity is the sum of the $S$ relative yields, or Relative Yield Total, RYT (de Wit 1970):

$$
R Y T=\sum_{i=1}^{S} y_{i}
$$

This index allows comparing community productivity on a relative basis. For instance, in the agricultural sciences, the $R Y T$ is often used to quantify the overyielding of diverse plant mixtures relative to plant monocultures in studies of biodiversity effects on ecosystem function. A RYT $>1$ implies species complementarity and that species partition the niche so as to reduce inter-specific competition (Vandermeer 1989, Loreau and Hector 2001). This, in turn, implies that the yield performance will be better in polyculture than in monoculture, a phenomenon termed as overyielding (Vandermeer 1989). The 
$R Y T$ divided by $S$ is equal to the Mean Relative Yield, $M R Y$, a second measure of community productivity:

$M R Y=R Y T / S$

Next, we can define the mean inter-specific competition parameter, $a$, as the mean over the off-diagonal elements of the matrix $\left\{a_{i j}\right\}$ :

$$
a=\overline{a_{i \neq j}}
$$

Recently derived analytical approximate expressions for the RYT and MRY as functions of this mean competition parameter $a$ and the species richness $S$ are provided by (Fort 2018, Fort and Segura 2018):

$$
R Y T(a, S) \simeq \frac{S}{1+(S-1) a},
$$

$\operatorname{MRY}(a, S) \simeq \frac{1}{1+(S-1) a}$.

To test formulae (5) and (6), a set of 25 experimental studies with $S>2$ that measured all the species yields was collected from the literature (Fort 2018). Some of these experiments were completed in laboratory and others in the field under natural conditions (see Table 1 for a comprehensive list of experiments used and their references). For $S>8$, I could not find an experiment in which the totality of the required treatments was carried out but only a percentage $f_{e}$ of the them. Thus for 12 out of these 25 experiments $f_{e}<100 \%$ (Table 1 ). The proposed method aims at providing $R Y T$ precisely for cases in which we have incomplete knowledge of the matrix $\left[a_{i j}\right]$. $^{1}$

Table 1, which is a modified version from the one published in Fort (2018), summarizes and compares the theoretical and experimental results.

The relative error between the theoretical approximate formula (5) and the empirically observed $R Y T, \varepsilon_{T E}$, is $10 \%$ or less for 8 out of 25 (32\%) of the experiments (Table 1). This accuracy is remarkable since the experimental SE for such quantities are in general greater than $\varepsilon_{T E}$ (in the interval $2-43 \%$, mean $=21 \%$ ). Furthermore, whenever $\varepsilon_{T E}$ is large $S E$ is also large, and thus the failure of eq. (5) can be explained by a low experimental precision.

Also notice that as $S$ increases the fraction $f_{e}$ of the $S \times(S+1) / 2$ experiments required to estimate the experimental quantities also decreases.

The dependence of the total yield with the species richness and the intensity of competition

Figure 1a shows the experimental and theoretical RYT vs. $S$ for the 25 experiments listed in Table 1 . We can see that the $R Y T$ predicted by eq.(5) for the minimum and maximum (a)

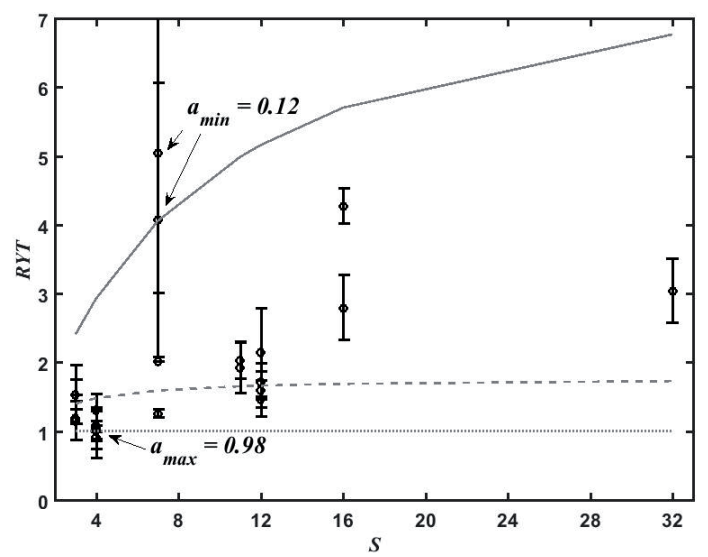

(b)

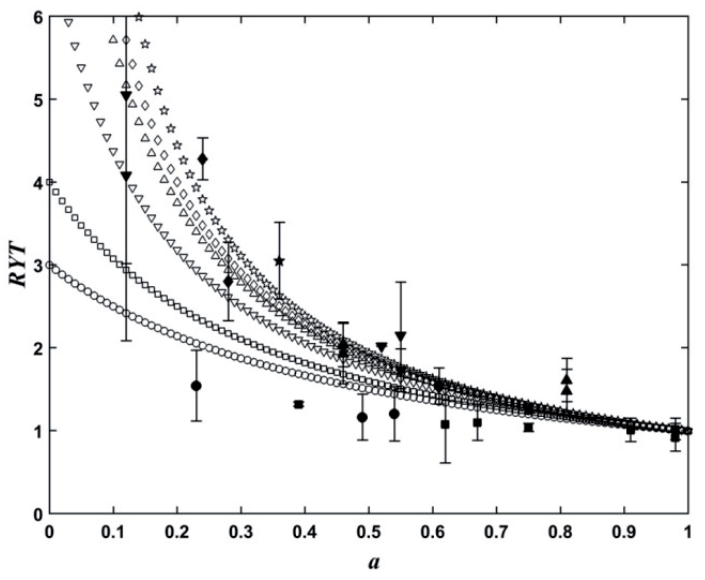

Figure 1.a. The dependence of $R Y T$ on species richness $S$. Experimental values (black circles with error bars) and theoretical RYT predicted by eq. (5) for the minimum, mean \& maximum experimentally measured mean inter-specific competition parameter in Table 1, respectively, $a=0.12$ (gray line), $a=0.56$ (gray dashed line) and $a=0.98$ (gray dotted line). b: The dependence of $R Y T$ on the mean inter-specific competition parameter $a$. Experimental values (black symbols with their SE bars) and the corresponding theoretical $R Y T$ predicted by eq. (5) (open symbols curves): $S=3$ (circles), $S=4$ (squares), $S=7$ (triangles down), $S=12$ (triangles), $S=16$ (diamonds) and $S=32$ (stars).

experimentally measured mean inter-specific competition parameter in Table 1, respectively, $a=0.12$ (gray line) and $a=$ 0.98 (gray dotted line), both fall within the error bars of the corresponding experimental values (black circles). In addition we plotted the theoretical $R Y T$ curve for $a=0.56$ (gray dashed line) which is the mean value of parameter $a$ over the 25 experiments.

Notice that the RYT increases with $S$, a phenomenon which has been observed in many empirical studies and interpreted as niche partitioning (Cardinale et al. 2012). That is, different species can use resources in a complementary way and therefore more diverse communities will lead to a high-

1 The details of the recipe used in these cases for estimating the experimental average interspecific competition coefficient $a$ (to feed equation (5)) and the experimental $R Y T$ are explained in Fort (2018) 
Table 1. RYT predicted by eq. (5) vs. experimental values for 25 ecological communities across different taxa (mainly vascular plants, but also algae, insects and crustaceans). $S$ is the number of coexisting species; exp.and theo. denote, respectively, experimental and theoretical; $a$ is the measured mean interspecific competition coefficient; $R Y T$ is the relative yield total; $\varepsilon_{T E}$ is the relative difference of theo. $R Y T$ respect to exp. $R Y T$ as $\% ; \mathrm{SE}$ is the standard error as $\%$ and $f_{e}$ is the percentage of the total $S \times(S+1) / 2$ experiments which were used to estimate the experimental quantities. For references to the experimental studies, see Fort (2018).

\begin{tabular}{|c|c|c|c|c|c|c|c|c|c|}
\hline Taxon & $\begin{array}{l}\text { Community (site, year, treatment, } \\
\text { etc) }\end{array}$ & $S$ & exp. $a$ & $\begin{array}{l}\text { exp. } \\
R Y T\end{array}$ & $\begin{array}{l}\text { theo. } \\
R Y T\end{array}$ & $\varepsilon_{T E} \%$ & exp.SE \% & $f_{e} \%$ & Note \\
\hline Algae & Santa Catalina Is., California & 3 & 0.61 & 1.53 & 1.35 & 12.4 & 14.1 & 100 & \\
\hline Plants & Grassland, San Jose, Ca. 1998 & 3 & 0.23 & 1.54 & 2.06 & 33.4 & 27.6 & 100 & [a] \\
\hline \multirow[t]{3}{*}{ Plants } & Iowa, 2003-2005, 1 cut & & & & & & & & {$[\mathrm{b}]$} \\
\hline & species mixture: IBF-IW-EG & 3 & 0.54 & 1.20 & 1.44 & 19.9 & 27.2 & 100 & \\
\hline & species mixture: IBF-SW-EG & 3 & 0.49 & 1.16 & 1.51 & 29.9 & 23.9 & 100 & \\
\hline Algae & continuous culture systems & 4 & 0.75 & 1.04 & 1.23 & 18.1 & $>4.7$ & 100 & \\
\hline Crustaceans & Lab. microcosms & 4 & 0.39 & 1.32 & 1.85 & 39.4 & $>2.0$ & 100 & {$[c]$} \\
\hline Plants & Random distribution experim. & 4 & 0.98 & 0.92 & 1.02 & 11.0 & 18.1 & 100 & \\
\hline \multirow[t]{4}{*}{ Plants } & Pastures, British Columbia & & & & & & & & \\
\hline & 1939 pasture & 4 & 0.67 & 1.10 & 1.33 & 21.5 & 19.8 & 100 & [d] \\
\hline & 1958 pasture & 4 & 0.98 & 1.02 & 1.01 & 1.0 & 12.7 & 100 & \\
\hline & 1977 pasture & 4 & 0.91 & 1.01 & 1.07 & 6.6 & 14.0 & 100 & \\
\hline Protozoa & Cultures \& lab. experiments & 4 & 0.62 & 1.08 & 1.62 & 49.6 & 43.5 & 100 & \\
\hline Plants & Otago lawn, NZ & 7 & 0.75 & 1.27 & 1.24 & 2.5 & 4.5 & 100 & \\
\hline Plants & Shoreline of Axe Lake, Ontario & 7 & 0.52 & 2.02 & 1.74 & 14.1 & NA & 75 & \\
\hline \multirow[t]{10}{*}{ Plants } & BIODEPTH exp., year 3 & & & & & & & & \\
\hline & Sweden, blok R6P009 & 7 & 0.12 & 5.05 & 4.56 & 9.6 & 40.3 & 35.7 & [e] \\
\hline & Sweden, blok R6P010 & 7 & 0.12 & 4.08 & 4.56 & 11.8 & 48.9 & 35.7 & [e] \\
\hline & Silwood, UK, mix.nest 233 & 11 & 0.46 & 2.04 & 1.97 & 3.3 & 13.2 & 16.7 & {$[\mathrm{e}],[\mathrm{f}],[\mathrm{g}]$} \\
\hline & Silwood, UK, mix.nest 234 & 11 & 0.46 & 1.93 & 1.97 & 1.9 & 18.9 & 16.7 & {$[\mathrm{e}],[\mathrm{f}],[\mathrm{g}]$} \\
\hline & Germany, mix.nest 28 & 12 & 0.55 & 2.15 & 1.71 & 20.1 & 29.9 & 24.4 & {$[\mathrm{~h}],[\mathrm{i}]$} \\
\hline & Germany, mix.nest 29 & 12 & 0.55 & 1.73 & 1.71 & 0.8 & 14.9 & 24.4 & {$[\mathrm{~h}],[\mathrm{i}]$} \\
\hline & Sheffield, UK, block S7P012 & 12 & 0.81 & 1.61 & 1.21 & 24.5 & 16.2 & 21.8 & {$[\mathrm{~h}]$} \\
\hline & Sheffield, UK, block S7P023 & 12 & 0.81 & 1.48 & 1.21 & 18.0 & 17.6 & 21.8 & \\
\hline & Sheffield, UK, block S7P023 & 12 & 0.81 & 1.61 & 1.21 & 24.8 & 16.2 & 21.8 & \\
\hline Plants & Cedar Creek E120, year 2003 & 16 & 0.24 & 4.28 & 3.73 & 12.9 & 5.9 & 30.1 & \\
\hline Plants & Jena exp., year 2007 & 16 & 0.28 & 2.80 & 3.06 & 9.5 & 16.9 & 17.6 & [j] \\
\hline
\end{tabular}

[a] More aggregate data: for functional groups rather than individual species.

[b] Averages over two different places and across the three years.

[c] $K$ obtained from the removal of 2 species rather than $S-1=3$.

[d] exp. $R Y T$ is a lower bound since there are other six additional species whose relative yields were not measured.

[e] The number of coexisting species $S$ was smaller than the number of seeded species (12) in this plot.

[f] In mix.nest 233 (234) of Silwood all except one (all) of the 11 relative yields y of the polyculture are available.

[g] In spite of many positive interactions the $\%$ error is small.

$[\mathrm{h}]$ a was computed from mean biomass densities for mono \& bicultures: $\bar{b}=\bar{B} \beta \bar{K} M, a=(1-\bar{b}) / \bar{b}$.

[i] The experimental $R Y T$ was computed approximately as $S \bar{Y} / \bar{K} M$.

[j] The experimental $R Y T$ was computed approximately by averaging $\Sigma_{i} Y_{i} / \bar{K} M$.

er RYT. However it was noticed that in spite of widespread claims about the role of biodiversity and ecosystem functioning relationships, researchers have rarely provided any direct quantitative evidence that niche partitioning is responsible for higher productivity in mixtures. The $R Y T>1$ for all except one of the experiments considered here (see Table 1), is a 
clear quantitative evidence of such species complementarity (de Wit 1970, Loreau and Hector 2001). For fixed mean competition parameter $a$, equation (5) becomes a non-linear saturating relationship between diversity and yield. A novelty here is that we provide the specific functional dependency for $R Y T$ with $S$ : an hyperbolic function (gray curves in Fig. 1.a). As expected, notice that, for fixed $S$, the RYT (Fig. 1.b) decreases with $a$.

\section{Applicability}

Regarding applications, the derived $R Y T$ approximate formula can be useful in biodiversity-ecosystem functioning $(\mathrm{BEF})$ research in cases in which measuring directly the RYT is difficult. For example, the artificial communities of BIODEPTH experiments (Hector et al. 2010), designed to measure this relation between species richness and productivity in plant communities. The maintenance of mixtures of many different coexisting species in this kind of experiments was a difficult task, implying intensive management such as hand weeding to maintain low diversity plantings and exclude unplanted species. The formula for the RYT may also be used to estimate the effect of removing existing species or adding foreign species to a community through the changes in the mean intensity of the interspecific competition they produce.

\section{Parsimonious modelling: proportionality between the available data and the means.}

For the reasons explained at the beginning, it seems a sensible approach to use the smallest possible number of parameters and, more importantly, parameters that can be practically estimated from empirical data, either experiments or field work. In particular, the incomplete knowledge of model parameters implies the necessity of an aggregation strategy since we cannot work with variables for individual species. One way to aggregate variables is to look for global community quantities - i.e., for the whole community of interacting species. Therefore here we contrasted predictions for the $R Y T$ and $M R Y$ using simple formulas we recently derived (Fort 2018) in terms of the average competition strength $a$, estimated from the yields for a reduced sample experimental treatments, against empirical results for a wide variety of communities (from protozoa to mammals). This aggregation procedure could be complemented with others like working with size-classes (Fort and Mungan 2015), or functional groups (Segura et al. 2013), or abundance-generalist classifications (Fort et al. 2016) etc. To conclude, the approximation we consider here seems to be good enough for many practical purposes and is proportional to the problem that it seeks to solve.

Acknowledgments. Work supported in part by ANII-Uruguay (SNI and project ERANET-LACR\&I2016-1005422).

\section{References}

Cardinale, B.J. et al. 2012.Biodiversity loss and its impact on humanity. Nature 486:59-67.

Fort, H. 2018. Quantitative predictions from competition theory with an incomplete knowledge of model parameters tested against experiments across diverse taxa. Ecol. Model. 368:104-110.

Fort, H., Dieguez, F., Halty, V. and Soares-Lima, J.M. 2017. Two examples of application of ecological modeling to agricultural production: Extensive livestock farming and overyielding in grassland mixtures. Ecol. Model. 357:23-34.

Fort, H. and Mungan, M. 2015. Predicting abundances of plants and pollinators using a simple compartmental mutualistic model. Proc. Royal Soc. B282, 1808.

Fort, H. and Segura, A. 2018. Competition across diverse taxa: quantitative integration of theory and empirical research using global indices of competition. Oikos, DOI: 10.1111/oik.04756.

Fort, H., Vázquez, D.P. and Lan, B.L. 2016. Abundance and generalisation in mutualistic networks: solving the chicken-and-egg dilemma. Ecol. Lett. 19:4-11.

Halty, V., Valdés, M., Tejera, M., Picasso, V. and Fort, H. 2017. Modelling plant interspecific interactions from experiments of perennial crop mixtures to predict optimal combinations. Ecol. Appl. 27:2277-2289.

Hector, A. et al. 2010. The data sets used in the paper with detailed descriptions. Ecological Archives E091-155-S1, Suppl. to Ecology 91:2213-2220. http://www.esapubs.org/archive/ecol/E091/155/

Hubbel, S.P. 2002. The Unified Neutral Theory of Biodiversity and Biogeography. Princeton Univ. Press, Princeton, NJ.

Loreau, M. and Hector, A. 2001 Partitioning selection and complementarity in biodiversity experiments. Nature 412:72-76.

Lotka, A.J. 1925. Elements of Physical Biology. Williams and Wilkins, Baltimore.

Segura, A.M., Kruk, C., Calliari, D. and Fort, H. 2013. Use of a morphology-based functional approach to model phytoplankton community succession in a shallow subtropical lake. Freshwater Biol. 58:504-512.

Vandermeer, J.H. 1989 The Ecology of Intercropping. Cambridge University Press, Cambridge.

Volterra, V. 1926. Fluctuations in the abundance of a species considered mathematically. Nature 118:558-560.

de Wit, C.T. 1970. On the modelling of competitive phenomena. Proc. Adv. Study Inst. Dynamics Numbers Popul. Oosterbeek, 1970:269-281.

Received November 30, 2017 Revised April 5, 2018 Accepted April 13, 2018 